$\pm 56.4 \mathrm{pg} / \mathrm{ml}), \mathrm{CT}(4855 \pm 268 \mathrm{pM})$ and $1.25-2 \mathrm{OH}$-vit D $(46.8 \pm 7.4 \mathrm{pg} / \mathrm{ml})$ showed high values. After treatment the calcium-treated women showed a significant decrease of AP (15.7 $\pm 3.3 \mathrm{U} / \mathrm{l}), \mathrm{PTH}(35.8 \pm 25.8 \mathrm{pg} / \mathrm{ml}), \mathrm{CT}(2490 \pm 1498 \mathrm{pM})$ and $1,25-2 \mathrm{OH}$-vitD $(38.0 \pm 8.0 \mathrm{pg} / \mathrm{ml})$ while the other parameters remained without significant changes. The placebo-group showed increments of AP, PTH, CT and 1.25-2OH-vitD.

Conclusions In pregnant women with low dietary $\mathrm{Ca}$ intake a parathyroid hyperfunction is present at least at the 20th week of pregnancy, a situation reverted by calcium supplement. In contrast to other studies IGF-1 did not show a systematic increase.

\section{HP0016 FAMILIAL RISK TO OSTEOPOROSIS IN THE IRISH POPULATION}

${ }^{1} \mathrm{M}$ Daly, ${ }^{2} \mathrm{~K}$ Quane, ${ }^{2} \mathrm{~F}$ Wynne, ${ }^{2} \mathrm{~F}$ Drummond, ${ }^{1} \mathrm{MG}$ Molloy. ${ }^{1}$ Rheumatology; ${ }^{2}$ Medicine, Cork University Hospital, Cork, Ireland

10.1136/annrheumdis-2001.1291

Osteoporosis is a disease characterised by a low bone mineral density (BMD) and microarchitectural deterioration of bone tissue leading to fractures, especially of the hip and spine. It is a major public health problem of western societies that affects the elderly population. Both environmental and genetic factors have been evoked as determinants of peak bone mass, but the major determinant of BMD is heritability. Families were recruited as part of an ongoing study to identify susceptibility genes to low BMD. Osteoporotic and osteopenic women were identified retrospectively from records at the Bone Densitometry Unit of Cork University Hospital. Individuals with a disease or on medications known to influence calcium metabolism were excluded. Participants were requested to nominate both female and male relatives for bone densitometry measurement. Relatives over 40 years of age, with a history of fracture or dowager's hump were preferentially selected for inclusion in the study. Of the 220 families who participated in the study to date, relative pairs with low BMD were found in 152 families. There was a total of 525 low BMD pairs among the 152 families, of whom 310 were sibpairs. Our study will help to increase public awareness in Ireland of the familial risk of osteoporosis.

\section{Fibromyalgia}

\section{HP0017 EVALUATING THE EFFECTIVENESS OF A FIBROMYALGIA EXERCISE PROGRAMME}

FM Russell, PA McEleny, DA Marshall. Department of Rheumatology, Inverclyde Royal Hospital, Greenock, UK

10.1136/annrheumdis-2001.1292

Background Exercise has been identified as the most effective intervention in the management of Fibromyalgia (FM), however, patients often report difficulty in initiating and maintaining a regular exercise programme., ${ }^{1,2}$ In 1996 a FM programme was developed at our hospital. Patients attend for 6 weeks and follow a progressive exercise programme of land-based exercise and hydrotherapy. The initial emphasis is on conditioning and stretching as preparation for increased activity. Patients are then encouraged to develop their own home exercise/activity programme, which they should continue on completion of our programme.

Method 50 consecutive patients with FM were diagnosed by Consultant Rheumatologist using the ACR criteria for $\mathrm{FM}^{3}$ Female patients 39 (78\%), male patients 11 (22\%), age range $28-78$ (mean 51.8 years). Symptom duration was as follows; $<1$ Year 11.1\%; 1-5 Years 50.0\%; 6-10 Years 16.7\%; > 10 Years 5.5\%; Patient Unsure 16.7\%. Time since diagnosis was as follows; Less Than 1 Year 55.6\%; 1-5 Years 38.9\%; 6-10 Years $5.5 \%$. Patients completed a series of validated questionnaires prior to participation on the programme and again at 6 weeks. The Nottingham Health Profile, Health Assessment Questionnaire and Hospital Anxiety Depression Scale were used thus assessing the major areas affected by FM - quality of life, mood, function and pain.

Results A third of patients reported improvements in mood and in some measures of global health specifically Physical Function and Social interaction. Generally pain did not significantly improve. However it should be remembered that this programme is designed as an initiation to exercise. Much of the work previously reported regarding the benefits of exercise entailed aerobic exercise with most studies having high drop out rates. ${ }^{4,5}$ Our programme introduced patients to gentle levels of exercise and progressed to more intensive work. The limitations of a 6-week programme mean we cannot progress to the aerobic levels where it has been demonstrated that pain and fatigue levels can be altered. The next stage of our project is to develop a community-based exercise programme. This combined approach of a gentle initiation followed by long-term exercise will be assessed at 6 and 12 months.

Conclusion We believe that this collaboration is innovative and has great potential in offering significant health improvements to Fibromyalgia patients.

\section{REFERENCES}

1 Waylonis MD, Perkin RH. Post-traumatic fibromyalgia - a long-term follow- up. Am J Phys Med. 1996;73:404-12

2 Horven Wigers $S$, Stiles TC, Vogel PA. Effects of aerobic exercise versus stress management treatment in Fibromyalgia. A 4-5 year prospective study. Scand J Rheumatol. 1996;25:77-86

3 Wolfe F, Smythe HA, Yunus MB. The American College of Rheumatology 1990 criteria for the classification of Fibromyalgia. Report of the Multi-centre Criteria Committee. Arthritis Rheum. 1990;33:160-72

4 Martin L, Nutting A, Mcintosh BR, Edworthy SW, Butterwick D. An exercise programme in the treatment of Fibromyalgia. J Rheumatol. 1994;23(6):1050-3

\section{Orthopedics, sports medicine/soft tissue rheumatism}

\section{HP0018 THE DEVELOPMENT OF AN INTEGRATED CARE PATHWAY FOR THE MANAGEMENT OF ANTERIOR KNEE PAIN IN CORNWALL}

PJ Pender. Rheumatology, Royal Cornwall Hospital, Truro, UK

10.1136/annrheumdis-2001.1293

Patients with anterior knee pain (AKP) are waiting long periods to see Orthopaedic surgeons for expert advice, few are considered for surgery, most are referred for physiotherapy. This expensive use of a Consultants time increases orthopaedic waiting lists and often delays patients referral to Physiotherapy. 
Objectives To develop management strategies by reviewing evidence base and current practice to increase numbers of patients seen in an orthopaedic clinic and improve the cost effectiveness and quality of health care.

Methods Evidence from literature and current practice by expert opinion was collated and analysed; from which evidence-based guidelines for the physiotherapy management of anterior knee pain (AKP) were formulated. Forty patients who presenting to an Orthopaedic clinic with AKP were assessed by a Clinical Specialist Physiotherapist and offered physiotherapy treatment, according to the guidelines. Management and treatment outcomes were audited.

Results 40 extra new patients were assessed in an orthopaedic clinic over 8 months (Age range 11-53 years, mean 25 years, symptom duration ranges 6 months-16 years). 57\% of the patients had not received any previous physiotherapy for their AKP. 3 patients were not offered physiotherapy and 3 were transferred to other physiotherapy departments. 5 did not attend a full course of treatment. 29 completed their treatment. Treatment duration ranged from 4-32 weeks, with a mean of 15 weeks. The number of treatments per patient ranged from 3-12 with a mean of 6 .

Treatment was tailored to the individual patient and consisted of education and exercises aimed at improving postural alignment, balance and the timing of the vastus medialis obliquis, gluteal and abdominal muscles. Manual therapy techniques and stretches were used to elongate tight structures e.g. hamstring, gastrocneiumi, muscles and ilio-tibial bands and lateral retinaculum. Patello-femoral taping was used in $26 \%$ of cases, trigger point acupuncture in 33\% of cases to provide some pain relief during their course of rehabilitation and $7 \%$ were provided with insoles.

At final assessment of the 29 patients that completed treatment, $52 \%$ stated that they were pain free. $22 \%$ stated that they were between $70-90 \%$ better. $15 \%$ stated that they were between $50-70 \%$ better. $7 \%$ were unresponsive to treatment. A proposal for an integrated care pathway for the management of AKP has been developed from these results and presented to the physiotherapists in Cornwall.

Conclusion Current literature and expert opinion advocates conservative management of anterior knee pain in the form of physiotherapy targeted at reconditioning lower limb musculature to improve patellofemoral biomechanics over surgical intervention. The results of this project support the literature but many patients are not being referred appropriately to Physiotherapists. an integrated care pathway for AKP has the potiential to create a co-ordinated cost effective approach to patient care.

\section{Pain: dealing with pain and pain management}

\section{HP0019 PHYSIOTHERAPIST/STUDENT FOR THE DEGREE OF MASTER OF SCIENCE}

TS Soyseth, V Soyseth. Department of Respiratory Medicine, National University Hospital, Oslo, Norway

\subsection{6/annrheumdis-2001.1294}

An investigation on the effect of education or education in combination with physical training on pain experience in women with osteoporosis.
Introduction Pain and fear of fracture are very common among patients with osteoporosis reducing their activity of daily living and quality of life. We have investigated the effect of education or education in combination with physical training in women with osteoporosis.

Methods Women older than 60 years with osteoporosis $(\mathrm{N}=$ 49) were enrolled in the study. All were able to walk indoor without technical support. Bone density was assessed using a DXA-machine.

All were hospitalised for five days in a hospital of rheumatology. They were casually put into two different treatment programs:

- Education ( $\mathrm{n}=26$ )

- Education in combination with physical train $(n=23)$

The education program was provided by a team of professional health workers and had a multifactorial approach to problems experienced by patients with osteoporosis. The training program was conducted by two physiotherapists.

Health was assessed by SF-36 questionnaire at baseline, and then after 3 and 12 months.

Results A multivariate analysis for repeated measurements showed an improvement of bodily pain score of 7.4 (95\% CI 2.7-12.1) after 3 months and 9.8 (95\% CI 4.2-15.4) after 1 year compared with baseline. There were no significant differences between the groups. Physical activity and social function were also statistical improved at 3 month, but the improvement did not persist for the complete follow-up period.

Conclusion Participation a professional education program reduces the pain experience in women with osteoporosis, and the improvement appear to persist for at least one year. This result needs confirmation by further studies.

\section{HP0020 PAIN TREATMENT OF THE RHEUMATIC DISORDERS WITH ELECTRIC THERAPY}

NK Basic, M Basic, D Masatovic. Rheumatology, Clinic for Physical Medicine and Rehabilitation, Tuzla, Bosnia and Herzegovina

10.1136/annrheumdis-2001.1295

Pain relief is one of the most important assignments of physical therapy (PT) and for that purpose different procedures have been used. Their advantage is that they have no side effects, which is very important for rheumatic patients, who are forced to take NSAIDs for a very long period.

The aim of this work was to investigate effects of different electric procedures on pain and joint swelling of rheumatic patients.

Material and methods The investigation was conducted on 40 patients with knee osteoarthritis and rheumatoid arthritis. Every knee was treated with different electric procedure. Mean age of patients was 54.6 years (SD 8.9), and mean duration of the illness was 12.1 years (SD 8.7). Interferrent, modulated and galvanic currents have been used. Treatment lasted for four weeks and in that period every patient received 20 therapies. Pain degree has been evaluated according to analogue visual scale VAS (0-100) before and after the treatment. At the end of the treatment patients and physicians have given their evaluation of the PT effect separately with grades from 1 to 5 (1- poor effect and 5 - excellent effect).

Results All three currents had good analgesic effect and there was a statistically significant difference in the pain evaluation 